\title{
Caracterización físico-química y biológica del suelo cultivado con maíz en sistemas convencional, agroecológico y mixto en la Frailesca, Chiapas Physico-chemical and biological characteristics of the soil cultured with maize in conventional, agroecological and mixed systems in the Frailesca, Chiapas
}

\author{
Franklin B. Martínez-Aguilar ${ }^{1}$ (i), Francisco Guevara-Hernández ${ }^{2}$ (i),

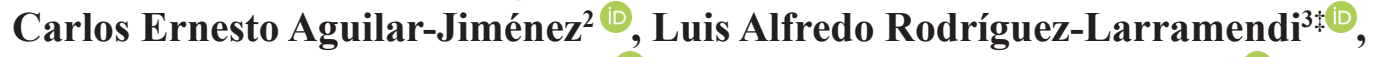 \\ Mariela Beatriz Reyes-Sosa ${ }^{4}$ (i) y Manuel Alejandro La O-Arias ${ }^{2}$ (i)
}

\begin{abstract}
${ }^{1}$ Estudiante de Doctorado en Ciencias Agropecuarias y Sustentabilidad (UNACH) $;{ }^{2}$ Investigador de la Facultad de Ciencias Agronómicas (UNACH); ${ }^{4}$ Facultad de Ciencias Agronómicas, CONACYT-UNACH, Universidad Autónoma de Chiapas (UNACH). Carretera Ocozocoautla-Villaflores km 84.5. 30470 Villaflores, Chiapas, México.

${ }^{3}$ Investigador de Facultad de Ingeniería, Sede Villa Corzo. Universidad de Ciencias y Artes de Chiapas (UNICACH). Carretera Villa Corzo-Monterrey km 3. 30520 Villa Corzo, Chiapas, México.

*Autor para correspondencia (alfredo.rodriguez@unicach.mx)
\end{abstract}

\section{RESUMEN}

Con el objetivo de caracterizar el suelo del agroecosistema maíz con los sistemas de producción convencional, agroecológico y mixto, para documentar e identificar estrategias que consideren los aspectos ambientales, sociales y económicos que conlleven a alternativas para la producción sostenible, se estudiaron las propiedades físico-químicas y biológicas de 35 muestras obtenidas en 28 ejidos de la región Frailesca, interpretadas con la Norma Oficial Mexicana NOM021-RECNAT-2000. Los resultados indican una acidez generalizada para los tres sistemas con un $\mathrm{pH}$ de 5.05, 5.25 y 5.24 , la capacidad intercambio catiónico es baja $\left(13.96,12.54\right.$, y $\left.14.95 \mathrm{cmol}(+) \mathrm{kg}^{-1}\right)$ y niveles medios de materia orgánica $(3.5,2.58$ y 3.45\%). Así mismo, los macronutrientes, como el fósforo se encuentra en niveles altos $\left(75.36,74.78\right.$ y $\left.45.05 \mathrm{mg} \mathrm{kg}^{-1}\right)$ y el potasio se encontró en niveles bajos (3.86, 3.12 y $\left.2.66 \mathrm{mg} \mathrm{kg}^{-1}\right)$ y los micronutrientes se encuentran en niveles medios como el hierro $\left(3.24,2.03\right.$ y $2.62 \mathrm{mg} \mathrm{kg}^{-1}$ ) y boro $\left(1.58,1.06\right.$ y $\left.1.37 \mathrm{mg} \mathrm{kg}^{-1}\right)$. Para el caso de la textura del suelo presentan suelos franco-arcillo-arenoso y franco-arenoso. Desde el punto de vista de las características biológicas del suelo, la macrofauna con el sistema agroecológico reportó mayor diversidad de seres vivos de acuerdo al índice de Shannon y

Cita recomendada:

Martínez-Aguilar, F. B., F. Guevara-Hernández, C. E. Aguilar-Jimenez, L. A. Rodríguez-Larramendi, M. B. Reyes-Sosa y M. A. La O-Arias. 2020. Caracterización físico-química y biológica del suelo cultivado con maíz en sistemas convencional, agroecológico y mixto en la Frailesca, Chiapas. Terra Latinoamericana 38: 871-881.

DOI: https://doi.org/10.28940/terra.v38i4.793
Weaver $\left(\mathrm{H}^{\prime}=1.121\right)$, destacan coleópteros, anélidos, himenópteros, isópteros y gasterópodos. En ese mismo sentido, para el caso de microorganismos sobresale en diversidad de microorganismos $\left(\mathrm{H}^{\prime}=1.121\right)$ reportando entomopatógenos (Beauveria bassiana y Metarhizium anisopliae), fijadores de nitrógeno (Rhizobium, Azotobacter y Azospirillum) y hongos patógenos como Rizhoctonia, Fusarium y Alternaria y el sistema con menor diversidad fue el convencional con $\mathrm{H}^{\prime}=0.789$. Bajo estas condiciones el suelo, es necesario una estrategia que permita aumentar la calidad y las prácticas de agricultura puedan ser sostenibles.

Palabras clave: macro y microorganismos, macronutrientes, micronutrientes.

\section{SUMMARY}

With the aim of characterizing the soil of the maize agroecosystem under conventional, agroecological and mixed production systems, in order to document and identify strategies that consider the environmental, social and economic aspects that lead to alternatives for sustainable production, the physico-chemical and biological properties of 35 samples obtained in 28 communa land areas of Frailesca region, interpreted with the Official Mexican Standard NOM-021- 
SEMARNAT-2000. The results indicate a generalized acidity for the three systems, with $\mathrm{pH}$ of 5.05, 5.25 and 5.24, respectively; the cation exchange capacity is low $\left(13.96,12.54\right.$ and $\left.14.95 \mathrm{cmol}(+) \mathrm{kg}^{-1}\right)$ and organic matter is at average levels $(3.5,2.58$ and $3.45 \%)$. Likewise, macronutrients are found in high levels, for instance, phosphorous reached 75.36, 74.78, and $45.05 \mathrm{mg} \mathrm{kg}^{-1}$ and potassium was found in low levels (3.86, 3.12, and $\left.2.66 \mathrm{mg} \mathrm{kg}^{-1}\right)$; micronutrients are found in average levels, such as Iron $(3.24,2.03$ and $2.62 \mathrm{mg} \mathrm{kg}^{-1}$ ) and Boron (1.58, 1.06 and $\left.1.37 \mathrm{mg} \mathrm{kg}^{-1}\right)$. In regard to soil texture, loam-clay-sandy and loamsandy soils were detected. According to the biological characteristics of the soil, the macro-fauna with the agroecological system reported the greatest biodiversity in terms of the Shannon and Weaver index $\left(\mathrm{H}^{\prime}=1.121\right)$, where coleopterans, annelids, hymenoptera, isoptera and gastropods were the most outstanding groups. For microorganisms, the greatest biodiversity $\left(\mathrm{H}^{\prime}=\right.$ 1.121) was detected in entomopathogens (Beauveria bassiana and Metarhizium anisopliae), nitrogen fixers (Rhizobium, Azotobacter and Azospirillum) and pathogenic fungi such as Rizhoctonia, Fusarium and Alternaria, while the system with smallest diversity was the conventional, with $\mathrm{H}^{\prime}=0.789$. Under these soil conditions, a strategy that allows to increase soil quality and perform sustainable agricultural practices is needed.

Index words: macro and microorganisms, macronutrients, micronutrients.

\section{INTRODUCCIÓN}

El suelo es un recurso fundamental para la producción de alimentos, sin embargo, se estima que a nivel nacional $87 \%$ de los suelos agrícolas presentan cierta degradación, debido a las diferentes prácticas de cultivo que provocan erosión, pérdida de nutrientes, salinidad y compactación (SEMARNAT, 2016). En ese sentido, Chiapas presenta un $79 \%$ y la región Frailesca $89 \%$ de suelos degradados por acciones naturales y antrópicas (Ortiz, 2019).

En 2017, el 45\% de la superficie cultivable en Chiapas se destinó a la producción de maíz, cuyo rendimiento promedio fue de $1.7 \mathrm{Mg} \mathrm{ha}^{-1}$ y una producción de 1147899 toneladas bajo condiciones de temporal (SIAP, 2018), convirtiéndolo así en el cultivo de mayor importancia en la alimentación en el estado.
La región Frailesca se ha caracterizado por ser una de las mayores productoras de maíz en el estado de Chiapas, con 59739 hectáreas sembradas en 2018, que produjeron alrededor de 193381 toneladas. De esa región, los municipios de Villaflores, La Concordia y Villa Corzo fueron los más productivos porque aportaron el mayor volumen de producción (181 241 toneladas) (SIAP, 2018). El rendimiento promedio de la región fue de $3.24 \mathrm{Mg} \mathrm{ha}^{-1}$, superior en $90.5 \%$ al promedio estatal, lo cual se debe principalmente al sistema convencional que se caracteriza por el uso de semillas mejoradas y cantidades elevadas de fertilizantes sintéticos, fundamentalmente nitrogenados, lo que implica un incremento en el costo de producción. Esto ha originado problemas de degradación física, química y biológica de suelos (López et al., 2019).

Actualmente, los suelos de la región han perdido su capacidad productiva debido a diferentes fenómenos naturales y antropogénicas como resultantes de la producción de maíz, sin importar la tecnología utilizada para producirlo. Esto ha ocasionado la disminución de los rendimientos, lo que exige cada vez más una mayor demanda de insumos externos para incrementar la producción. Bajo tal situación, el maíz se ha convertido en un cultivo poco rentable para la mayoría de los pequeños productores (Pulleman et al., 2008). A pesar de las evidencias sobre la pérdida de fertilidad del suelo y sus consecuencias, se desconoce la magnitud y la cobertura que podría tener este problema en la región (López et al., 2018).

El objetivo de esta investigación fue caracterizar el suelo del agroecosistema maíz bajo los sistemas de producción convencional, agroecológica y mixta, para documentar e identificar estrategias que consideren los aspectos ambientales, sociales y económicos que conlleven a alternativas para la producción sostenible.

\section{MATERIALES Y MÉTODOS}

El estudio se realizó durante el año 2018 en localidades de la región Frailesca, en parcelas de 35 productores de maíz distribuidos en 28 comunidades (Figura 1). Las parcelas de la forma de manejo convencional se localizan en zonas de orografía plana, a una altitud de $620 \mathrm{~m}$; mientras que las formas de manejo mixta y agroecológica se ubican entre 747 y $884 \mathrm{~m}$ de altitud respectivamente, en terrenos que fluctúan entre semiplanos a lomeríos, fundamentalmente sobre áreas con pendientes conocidas localmente como laderas. 


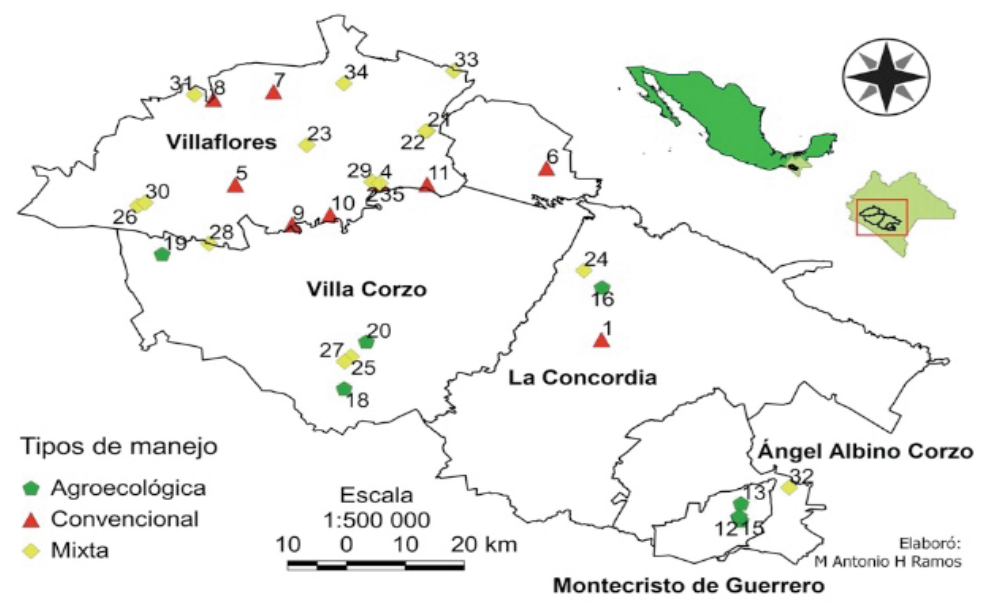

Figura 1. Localización de suelos estudiados bajo tres sistemas de manejo en la región Frailesca, Chiapas, México.

Figure 1. Location of soils studied under three management systems in Frailesca region, Chiapas, Mexico.

\section{Los Sistemas de Manejo del Agroecosistema}

De acuerdo con el uso e intensidad de la energía invertida en el agroecosistema maíz, en la región Frailesca existen diferentes variantes tecnológicas. Dichas variantes se han identificado como convencional, agroecológica y mixta, las cuales surgieron de una tipificación del uso de maquinaria agrícola, uso y cantidad de fertilizantes químicos, otros agroquímicos, prácticas de conservación de suelo y cantidad de superficie utilizada para la producción de maíz (Martínez et al., 2020), las cuales además son parte de un estudio más profundo sobre la sostenibilidad del agroecosistema maíz. Estas variantes también han sido estudiadas por Guevara et al. (2018).

\section{Caracterización Química, Física y Biológica de los Suelos}

Para conocer las características físicas, químicas y biológicas de los suelos, se realizó un muestreo en cada parcela, siguiendo el método de cinco de oros a una profundidad de $0-20 \mathrm{~cm}$, de acuerdo a lo recomendado por Mendoza y Espinosa (2017) porque es donde se desarrolla el mayor porcentaje del sistema radical del maíz. A partir de los muestreos, se obtuvieron 25 submuestras por parcela, se mezclaron y se obtuvo una muestra compuesta, las cuales fueron enviadas al laboratorio del Colegio Postgraduados Campus Montecillo para las determinaciones de materia orgánica
(\%), densidad aparente (Da), N-total (Kjeldahl), capacidad intercambio catiónico (CIC) $(\mathrm{KCl} 2 \mathrm{~N})$, textura (arcilla, limo y arena), $\mathrm{pH}\left(\mathrm{CaCO}_{2}\right), \mathrm{P}(\mathrm{Olsen})$, $\mathrm{K}, \mathrm{Ca}, \mathrm{Mg}\left(\mathrm{NH}_{4} \mathrm{O}\right.$ ac $\left.1 \mathrm{~N} \mathrm{pH}\right), \mathrm{Pb}, \mathrm{Zn}, \mathrm{Fe}$ (DTPA), $\mathrm{Cu}, \mathrm{Mn}, \mathrm{B}, \mathrm{Mo}(\mathrm{KCl} 1 \mathrm{~N})$ y Ni. La interpretación de las propiedades de los suelos se realizó de acuerdo con la Norma Oficial Mexicana NOM-021-RECNAT-2000 (SEMARNAT, 2002). Los ácidos húmicos, fúlvicos y carboxílicos, se detectaron a través de la técnica de la resonancia magnética (Mosquera et al., 2010; Rodríguez et al., 2016).

Para determinar la macrofauna del suelo se utilizó la metodología propuesta por Moreira et al. (2012) que consiste en muestrear cinco cuadrantes de $25 \mathrm{~m}^{2}$ (cinco de oros) en cada parcela. De cada cuadrante se obtuvieron cinco submuestras de $25 \times 25 \times 10 \mathrm{~cm}$. De cada submuestra se formó una muestra compuesta de $3 \mathrm{~kg}$ de suelo por parcela estudiada. Posteriormente se tamizó cada muestra de suelo con mallas de 2, 0.841 y $0.420 \mathrm{~mm}$. Se tomaron $50 \mathrm{~g}$ de suelo por malla para verificar con estereoscopio la existencia de seres vivos, los cuales fueron clasificados por Phyllum y orden.

Para estimar la diversidad de la macrofauna y microorganismos del suelo se utilizó el índice ( $\left.\mathrm{H}^{\prime}\right)$ de Shannon y Weaver (1949). Este índice contabiliza la cantidad de especies presentes en el área de estudio (riqueza de especies) y la cantidad relativa de individuos de cada especie (abundancia) (De la Rosa y Negrete, 2012). 


\section{Análisis Estadístico}

A todas las variables se les realizó una estadística descriptiva, para conocer las diferencias entre los sistemas de manejo (convencional, agroecológica y mixta), se utilizó el paquete estadístico SPSS versión 19. Además, se hizo un análisis de correspondencia múltiple entre las variables y las formas de manejo.

\section{RESULTADOS Y DISCUSIÓN}

Las características físicas y químicas de los suelos estudiados indican poca variabilidad de acuerdo con el coeficiente de variación (Cuadro 1), lo cual denota baja heterogeneidad en el efecto de los sistemas de manejo.

El contenido de nitrógeno resultó ser bajo en los sistemas convencional y mixto $(1.7 \%$ de $\mathrm{N})$ así como en el sistema agroecológico $(1.1 \% \mathrm{~N})$, según la Norma Oficial Mexicana: fertilidad, salinidad y clasificación de suelos, estudios, muestreo y análisis (SEMARNAT, 2002). Esto obedece a que el nitrógeno, por su alta movilidad, particularmente en condiciones tropicales, se encuentra hasta en un $80 \%$ en la biomasa de las plantas (Cepeda, 2016). Sin embargo, su disponibilidad depende de otros factores químico-biológicos, como la cantidad de residuo orgánico presente en el suelo y la acción de los microorganismos en la mineralización y humificación de la biomasa vegetal (Quiroga y Bono, 2012; Ortiz, 2019). Por ello, los productores tienen que suministrarle nitrógeno al maíz en forma de sulfato o nitrato de amonio en los tres sistemas de manejo.

Por otro lado, es preocupante la cantidad de plomo $(\mathrm{Pb})$ encontrado en el suelo, el cual, de acuerdo a la Norma Oficial Mexicana, por encima de $100 \mathrm{mg} \mathrm{kg}^{-1}$ de suelo es considerado peligroso. Los promedios encontrados fueron de $111.36,84$ y $90.20 \mathrm{mg} \mathrm{kg}^{-1}$ para los sistemas de manejo convencional, agroecológico y mixto, respectivamente. Esto significa que en el área de estudio los suelos cultivados con maíz bajo el sistema convencional la cantidad de plomo está por encima del limite señalado por la Norma Oficial Mexicana, mientras que para los otros dos sistemas, se encuentran cerca del límite peligroso. Esto puede tener diferentes causas, que van desde la aplicación indiscriminada

Cuadro 1. Estadígrafos de tendencia central y de dispersión de las características físicas y químicas de los suelos bajo tres sistemas de manejo.

Table 1. Statistics of central tendency and dispersion of the physical and chemical characteristics of the soils under three management systems.

\begin{tabular}{|c|c|c|c|c|c|c|c|}
\hline \multirow{2}{*}{ Variable } & \multicolumn{2}{|c|}{ Agroecológico } & \multicolumn{2}{|c|}{ Convencional } & \multicolumn{2}{|c|}{ Mixto } & \multirow{2}{*}{$\mathrm{CV}$} \\
\hline & Promedio & E.E. & Promedio & E.E. & Promedio & E.E. & \\
\hline & & & & & & & $\%$ \\
\hline Nitrógeno total (\%) & 1.10 & 0.035 & 1.7 & 0.032 & 1.7 & 0.028 & 0.074 \\
\hline Plomo (mg kg-1) & 84 & 22.57 & 111.36 & 19.46 & 90.2 & 15.22 & 7.54 \\
\hline $\mathrm{pH}$ & 5.05 & 0.149 & 5.25 & 0.134 & 5.24 & 0.115 & 0.196 \\
\hline M.O. (\%) & 3.5 & 0.580 & 2.58 & 0.52 & 3.45 & 0.46 & 4.46 \\
\hline $\mathrm{CIC}\left(\operatorname{cmol}(+) \mathrm{kg}^{-1}\right)$ & 13.96 & 3.879 & 12.54 & 3.509 & 14.95 & 3.11 & 3.12 \\
\hline $\mathrm{Da}\left(\mathrm{g} \mathrm{t}^{-1}\right)$ & 1.35 & 0.047 & 1.2 & 0.042 & 1.32 & 0.038 & 0.12 \\
\hline Arcilla (\%) & 21.78 & 3.659 & 19.62 & 3.310 & 25.45 & 2.934 & 2.308 \\
\hline Limo (\%) & 16.27 & 2.490 & 21.45 & 2.252 & 18.29 & 1.996 & 1.723 \\
\hline Arena (\%) & 61.78 & 5.339 & 58.93 & 4.829 & 56.26 & 4.280 & 2.092 \\
\hline Clase textural & $\begin{array}{l}\text { Franco- } \\
\text { arcillo- } \\
\text { arenoso }\end{array}$ & & $\begin{array}{l}\text { Franco- } \\
\text { arenoso }\end{array}$ & & $\begin{array}{l}\text { Franco- } \\
\text { arcillo- } \\
\text { arenoso }\end{array}$ & & \\
\hline
\end{tabular}

E.E. = error estándar; $\mathrm{CV}=$ coeficiente de variación.

E.E. $=$ standard error; $\mathrm{CV}=$ coefficient of variation. 
de agroquímicos como los fungicidas (Martí et al., 2009), herbicidas (UNL, 2010¹) e insecticidas (García y Rodríguez, 2012), hasta las diferentes actividades antropogénicas que se desarrollan en la periferia o proximidades del agroecosistema maíz, como la pavimentación de carreteras, uso de gasolina y materiales para soldaduras, entre otros (Yucra et al., 2008; García et al., 2012; Montiel e Ibrahim, 2015).

El pH del suelo en los tres sistemas de manejo fue de $5.05,5.24$ y 5.25 , que los clasifica como moderadamente ácidos, de acuerdo con la NOM-021RECNAT-2000. Sin embargo, el suelo manejado bajo el sistema agroecológico presentó el $\mathrm{pH}$ más bajo (5.05). Esto se relaciona con la cantidad de materia orgánica reportada para esta forma de manejo (3.5\%), pues al existir mayor cantidad de humus, contiene grupos reactivos de carboxilo, fenólicos e imídicos que son capaces de ligar iones de hidrógeno. Además, la cantidad de lluvia y la actividad de microorganismos son los principales factores de reacción del suelo, ya que su abundancia produce los principales efectos sobre el pH (Vieira et al., 1998; Jiménez-Ballesta y González-Quiñones, 2006; Alcántar et al., 2016). Estos resultados son muy similares a los reportados por López et al. (2019) quienes caracterizaron los suelos cultivados con maíz en Villaflores, Chiapas y encontraron $\mathrm{pH}$ 5.2, indicando una acidez generalizada entre los suelos estudiados. Sin embargo, el problema de un suelo ácido radica en la posible toxicidad que provocaría al precipitarse el aluminio y el manganeso causando deficiencias de calcio y magnesio (Espinosa y Molina, 1999; Tasistro, 2012²).

\section{Materia Orgánica}

La materia orgánica reportada se encuentra en un nivel medio, resaltando el sistema agroecológico con $3.5 \%$, la mixta $3.45 \%$ y convencional $2.58 \%$. Esto obedece a las prácticas de conservación del suelo en el sistema agroecológico, como el policultivo y la no quema de residuos orgánicos. En ese sentido, la incorporación de residuos orgánicos al suelo favorece la reproducción de microorganismos y macroorganismos, los cuales intervienen en la mineralización, lo que ocasiona cambios físicos, químicos y bioquímicos en el suelo y aumenta la disponibilidad de nutrientes a largo plazo (Jordán, 2006; Vallejo, 2013).
El análisis de correspondencia entre los sistemas de producción y los niveles de humificación de la materia orgánica no mostró diferencia significativa (Figura 2). Sin embargo, se asume que el sistema agroecológico está más vinculado con los ácidos húmicos y el convencional con los ácidos fúlvicos. Los ácidos fúlvicos están presentes cuando en el proceso de humificación de la materia orgánica se remueve el suelo, se oxigena y los microorganismos se activan para descomponer la materia orgánica (Labrador, 2001; Correa; 2013); por ello, el manejo convencional está relacionado con los ácidos fúlvicos, los cuales son más susceptibles al ataque microbiano y más soluble para las plantas (Primavesi, 1980; Pool et al., 2019). El manejo agroecológico se relaciona con los ácidos húmicos, el cual es más lento su proceso de degradación y no es soluble para las plantas, porque son polímeros de estructura compleja; es decir, el proceso de humificación en el manejo agroecológico es más lento.

Se determinó la asociación entre los sistemas de manejo y los diferentes ácidos orgánicos que se producen en el suelo por efectos químicos y biológicos (Figura 3). El sistema agroecológico presenta cuatro ácidos orgánicos (láctico, acético, fórmico y salicílico), los cuales son generados por las bacterias Rhizobium, Agrobacterium y Pseudomonas y sirven para la movilización del fósforo (Paredes y Espinosa, 2009). El ácido salicílico (AS) es importante cuando se asocia con el potasio para formar silicato de potasio, los cuales

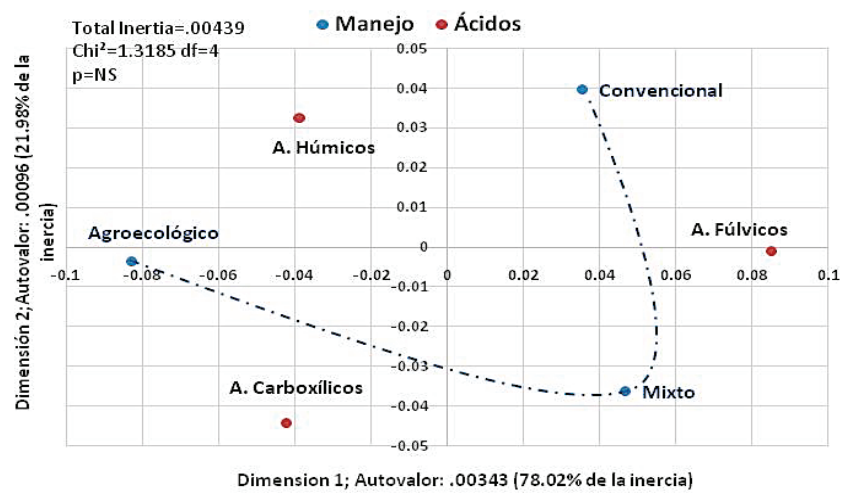

Figura 2. Análisis de correspondencia entre los sistemas de manejo y los ácidos orgánicos presentes en el suelo.

Figure 2. Correspondence analysis between management systems and organic acids in the soil.

\footnotetext{
${ }^{1}$ UNL (Universidad Nacional del Litoral). 2010. Informe acerca del grado de toxicidad del glifosato. Servicio altamente especializado a terceros. Santa Fé, Argentina.

${ }^{2}$ Tasistro, A. 2012. Acidez del suelo. Instituto Internacional de Nutrición Vegetal (IPNI). Notas de conferencia. 108p.
} 
dan a la planta mayor resistencia ante las enfermedades, al estrés hídrico y mayor resistencia del tallo al acame (Labrador, 2001).

La capacidad de intercambio catiónico (CIC) es baja para los tres sistemas de manejo (convencional 12.54, agroecológico 13.96 y mixto $\left.14.95 \mathrm{cmol}(+) \mathrm{kg}^{-1}\right)$ (SEMARNAT, 2002). Esto significa que los suelos en las formas de manejo tienen baja capacidad de intercambiar cationes con la solución del suelo (Primavesi, 1980). La CIC está relacionada con el pH, cantidad de materia orgánica y arcilla, porque entre más carga negativa tenga el suelo, mayor capacidad tendrá para retener e intercambiar cationes (JiménezBallesta y González-Quiñones, 2006; Estrada et al., 2017). Por ejemplo, los productores aplican cada vez más fertilizantes sintéticos para nutrir las plantas; sin embargo, su uso hace más ineficiente al sistema, ya que se lixivian cuando en la solución del suelo no hay suficientes aniones para retener los cationes, como lo aquí encontrado.

La textura de los suelos con respecto al sistema de manejo del agroecosistema indica que el convencional presenta un suelo con textura franco-arenosa, mientras que la mixta y agroecológica, poseen una clase textural franco-arcilloso-arenoso. Estas texturas obedecen a los años de uso de esos suelos y a que en la mayoría de los franco-arenosos, no existe un plan de conservación. Lo anterior se convierte en un problema, porque entre más arenoso sea un suelo menor será su capacidad de retención de agua (Camas et al., 2012). Además, si no realizan prácticas de conservación del suelo para disminuir la degradación, en poco tiempo el suelo perderá su capacidad productiva (Martínez et al., 2019). Sin embargo, estas clases texturales tienen condiciones apropiadas para el crecimiento del cultivo de maíz (López et al., 2019).

El fósforo $(\mathrm{P})$ encontrado en los suelos estudiados es alto $\left(75.36,74.78\right.$ y $45.05 \mathrm{mg} \mathrm{kg}^{-1}$ ) (SEMARNAT, 2002) para los tres sistemas de manejo (Figura 4). Estos valores son adecuados para el crecimiento de microorganismos, los cuales ayudan a la solubilización y movilización del $\mathrm{P}$ en el suelo, como las micorrizas que lo solubilizan y hacen disponible para las plantas (Rodríguez, 2005). Existe una diferencia de $35 \mathrm{mg}$ $\mathrm{kg}^{-1}$ entre el sistema de manejo mixto, el convencional y agroecológico, esto se atribuye a la roca madre (apatita) o a la cantidad de rocas fosfatadas que pueden encontrarse entre un lugar y otro (Bohn et al., 1993; Pool et al., 2000). Sin embargo, Camas et al. (2012) aseguran que la erosión del suelo en zonas de laderas es un factor para la pérdida de minerales como el fósforo proveniente de la materia orgánica; por ello,

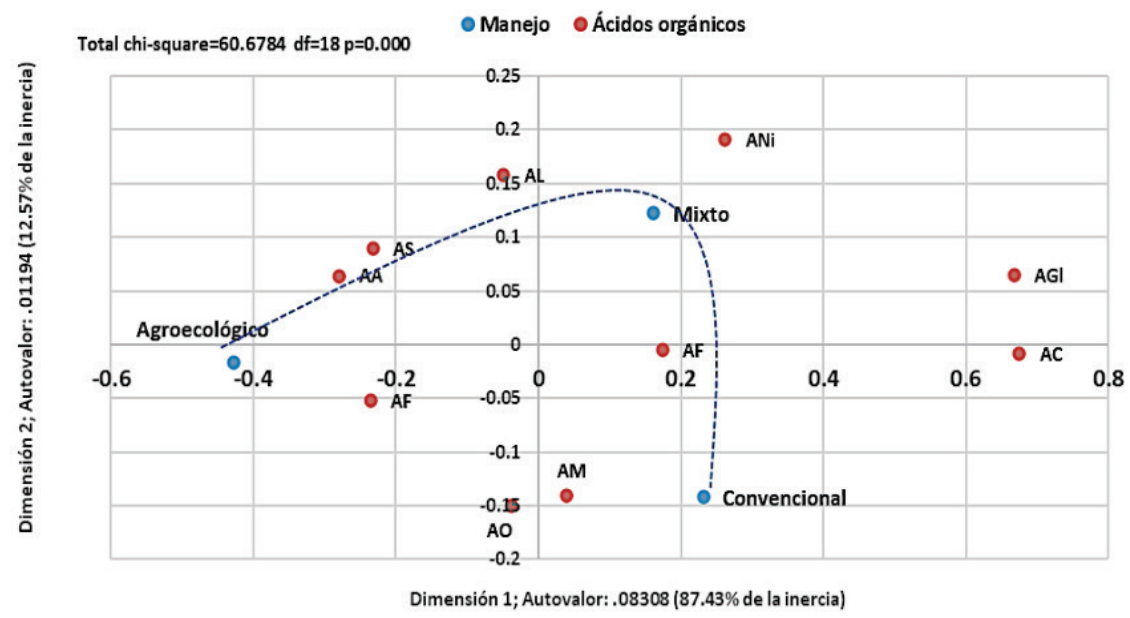

Figura 3. Análisis de correspondencia entre sistemas de manejo y ácidos carboxílicos encontrados en los suelos de la región Frailesca. $\mathrm{AF}$ = ácido fórmico; $\mathrm{AA}$ = ácido acético; $\mathrm{AS}$ = ácido salicílico; $\mathrm{AL}$ = ácido láctico; $\mathrm{Ani}=$ ácido nicótico; $\mathrm{AGl}=$ ácido glutámico; $\mathrm{AC}$ = ácido cítrico; $\mathrm{AM}=$ ácido málico; $\mathrm{AO}=$ ácido oxálico; $\mathrm{AF}$ = ácido fólico.

Figure 3. Correspondence analysis between management systems and carboxylic acids found in the soils of Frailesca region. $\mathrm{AF}=$ formic acid; $\mathrm{AA}=$ acetic acid; $\mathrm{AS}=$ salicylic acid; $\mathrm{AL}=$ lactic acid; $\mathrm{Ani}=$ nicotic acid; $\mathrm{AGl}=$ glutamic acid; $\mathrm{AC}=$ citric acid; $\mathrm{AM}$ $=$ malic acid $\mathrm{AO}=$ oxalic acid $\mathrm{AF}=$ folic acid. 
es importante la implementación de prácticas de conservación del suelo como curvas a nivel, terrazas, muros de contención y barreras vivas, para disminuir el efecto que tiene el agua al arrastrar el suelo a través de la escorrentía.

Para el potasio $(\mathrm{K})$ se reportaron niveles medios en los tres sistemas de manejo (convencional 3.86, agroecologico 3.12 y mixto $\left.2.66 \mathrm{mg} \mathrm{kg}^{-1}\right)$. Esto indica que el suelo suministra adecuadamente $\mathrm{K}$ para el crecimiento y desarrollo de la planta; ademas, el K es fundamental en el proceso de intercambio catiónico a nivel coloidal, es un activador de enzimas y se ubica en el sexto lugar de importancia en la composición de la materia seca en maíz con $9.2 \mathrm{~kg} \mathrm{Mg}^{-1}$ (Morón et al., 1996) y para el calcio (Ca) se obtuvieron valores medios (convencional 3.73, agroecolóogico $4.91 \mathrm{y}$ mixto $5.58 \mathrm{mg} \mathrm{kg}^{-1}$ ), con estos resultados las plantas no carecen de este nutriente y realizan todo su proceso celular. El Ca aporta $2.3 \mathrm{~kg}$ en una tonelada de materia seca de maíz (INPOFOS, 1988; Alcántar et al., 2016).

Los valores de magnesio $(\mathrm{Mg})$ registrados en los tres sistemas de manejo (convencional 15.32, agroecologico 9.22 y mixto $13.47 \mathrm{mg} \mathrm{kg}^{-1}$ ) fueron altos (Figura 4). Esto asegura que el reservorio del suelo cuente con suficiente Mg para proporcionarle a la planta de maíz lo necesario para su crecimiento. Por costumbre, los productores no aplican este mineral de manera adicional, para evitar el incremento en los costos de producción. Por ello, la gran mayoría solo aplican fertilizantes nitrogenados, provocando una acidificación del suelo y mayor oxidación de la materia orgánica (Wilson, 2017).

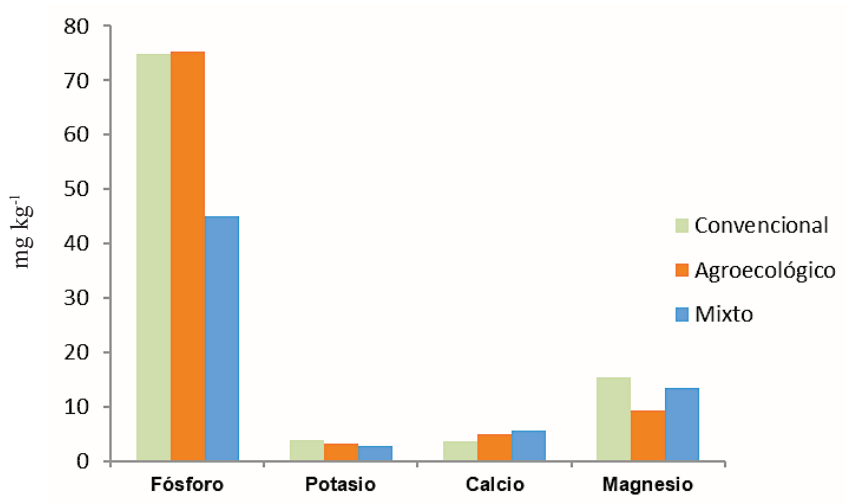

Figura 4. Contenido de macronutrientes primarios y secundarios con tres sistemas de manejo del suelo.

Figure 4. Content of primary and secondary macronutrients with three soil management systems.
Los micronutrientes ( $\mathrm{Zn}, \mathrm{Fe}, \mathrm{Cu}, \mathrm{Mn}, \mathrm{B}$, Mo y Ni) se encuentran en intervalos de adecuado a marginal en los tres sistemas de manejo (Figura 5). Esto indica que el suelo cuenta con estos micronutrientes esenciales para suminitrar a las plantas la cantidad que necesiten para su normal crecimiento y desarrollo. Sin embargo, en zonas tropicales, la cantidad de agua que precipita genera infiltración y escurrentías en el suelo, lo que provoca pérdida de dichos elementos por lixiviación y erosión (Prieto et al., 2013; Barrezueta et al., 2017). Por ello, la presencia de estos elementos está en función de la cantidad y la velocidad de descomposición de la materia orgánica (Silva, 2001).

Respecto a diversidad de microorganismos, el índice de Shannon y Weaver (H') indica que el sistema de manejo agroecológico presenta mayor diversidad y abundancia de microorganismos $\left(\mathrm{H}^{\prime}=1.254\right)$, seguido del mixto $\left(H^{\prime}=0.943\right)$ y convencional con $H^{\prime}=0.850$ (Figura 6) Los microorganismos encontrados fueron: fijadores de Nitrógeno (Rhizobium, Azotobacter, y Azospirillum), entomopatógenos (Beauveria bassiana y Metarhizium anisopliae), así como algunos patógenos de las raíces (Phytium-Rhizotocnia y Fusarium de la raíz) y de los tallos (Fusarium solani, Puccina y Alternaria). La diversidad y abundancia de microorganismos en el sistema agroecológico está relacionada con la cantidad de materia orgánica reportada para esta forma de manejo. Esto obedece a la incorporación de residuos orgánicos a través de la labranza de conservación, policultivos y la no quema, cuyas prácticas favorecen la vida en el suelo (Campbell, 2001; Cleveland y Liptin, 2007; Rodríguez et al., 2020).

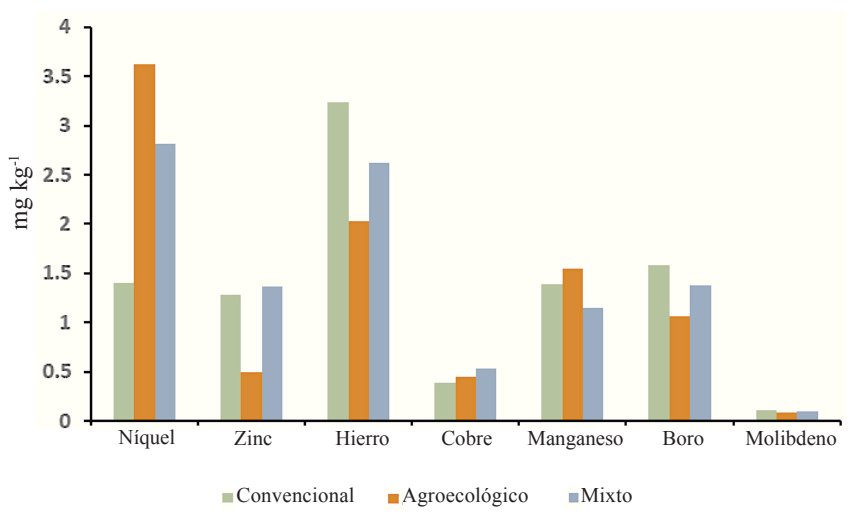

Figura 5. Contenido de micronutrientes en el suelo del agroecosistema maíz y tres sistemas de manejo.

Figure 5. Micronutrient content in the soil of maize agroecosystem and three management systems. 


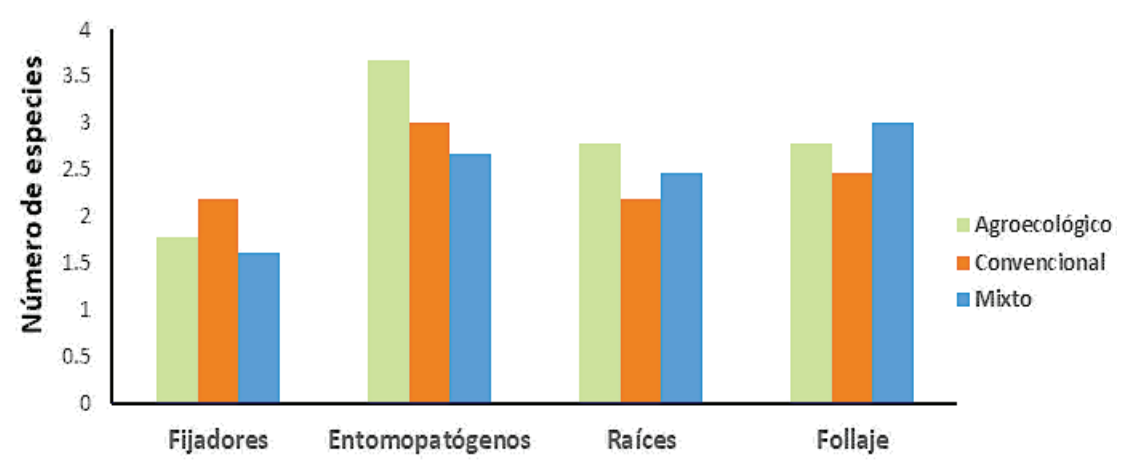

Figura 6. Número de especies de microorganismos benéficos y patógenos presentes en suelos de la región de la Frailesca con diferentes sistemas de manejo.

Figure 6. Number of species of beneficial and pathogenic microorganisms in soils of the Frailesca region with different management systems.

La macrofauna del suelo en el sistema agroecológico presentó mayor diversidad y abundancia, de acuerdo con el índice de Shannon y Weaver $\left(\mathrm{H}^{\prime}=1.121\right)$, destacando coleópteros, anélidos, himenópteros, isópteros y gasterópodos. Dichos macroorganismos son los encargados del proceso de descomposición de los residuos de cosecha y tienen efectos positivos sobre las propiedades del suelo. Estos resultados coinciden con Lal (1988) quién señala que la labranza de conservación y asociación de cultivos, incrementa la presencia de macroorganismos en el suelo, porque contribuyen a retener, humedad y son prácticas que realizan los productores en el sistema agroecológico.

En los sistemas de manejo convencional y mixto se observa baja diversidad de macroorganismos $\mathrm{H}^{\prime}=0.789$ y H' $=0.924$, respectivamente; esto podría deberse a la baja cantidad de materia orgánica aplicada en ambos sistemas de manejo y alto uso de agroquímicos, el cual afecta negativamente a los macroorganismos que cumplen con el proceso de humificación (Rendón et al., 2011).

\section{Características Antropogénicas y Naturales}

La zona de estudio se caracteriza por presentar condiciones antrópicas y naturales que condicionan el manejo del suelo, destacan los años de uso, implementación de policultivos, edad del productor y el tipo de maíz (Cuadro 2). Los productores bajo la forma de manejo convencional utilizan exclusivamente

Cuadro 2. Características antropogénicas y naturales del agroecosistema maíz con los sistemas de manejo.

Table 2. Anthropogenic and natural characteristics of the maize agroecosystem with management systems.

\begin{tabular}{|c|c|c|c|c|c|c|}
\hline \multirow{2}{*}{ Variable } & \multicolumn{2}{|c|}{ Agroecológico } & \multicolumn{2}{|c|}{ Convencional } & \multicolumn{2}{|c|}{ Mixto } \\
\hline & Promedio & E.E. & Promedio & E.E. & Promedio & E.E \\
\hline Edad del productor & 55.4 & 17.9 & 59.6 & 15.7 & 58.5 & 10.7 \\
\hline Uso del suelo bajo el sistema de manejo (años) & 14.6 & 12.1 & 16.7 & 16.3 & 26 & 16.0 \\
\hline Superficie de maíz & 2.6 & 2.9 & 5.7 & 8.2 & 2.5 & 1.5 \\
\hline Tipo de maíz cultivado & Maíz local ${ }^{\dagger}$ & & Híbrido & & Híbrido o Local & \\
\hline Tipo de propiedad & Ejidal & & Ejidal & & Ejidal / rentada & \\
\hline Cultivos asociados & $\mathrm{Si}$ & & No & & $\mathrm{Si}$ & \\
\hline
\end{tabular}

E.E.= error estándar. ${ }^{\dagger}$ Tipo de maíz que el productor cultiva por más de 3-5 años sin importar el origen (Guevara et al., 2019).

E.E. $=$ standard error. ${ }^{\dagger}$ Type of maize that the producer grows for more than 3-5 years regardless of its origin (Guevara et al., 2019). 
semilla híbrida; la agroecológica, 100\% utilizan maíces locales y la forma mixta utilizan híbridos o maíces locales. En ese tenor, los criterios de los productores para seleccionar una semilla local en esta región, está basada en las condiciones edafoclimáticas, organolépticas y que tengan usos múltiples (Delgado et al., 2018), lo que coincide para las formas no convencionales del cultivo de maíz, no centradas únicamente en los rendimientos agronómicos.

En el caso del manejo convencional, los suelos muestreados presentan en promedio 16.7 años de uso, mientras que los suelos con manejo agroecológico muestran 14.6 años y el manejo mixto 26 años, lo cual significa que se ha cultivado maíz en monocultivo de manera continua. Por ello, la baja fertilidad obedece a la no implementación de prácticas agroecológicas como labranza de conservación, uso de policultivos incorporación de abonos orgánicos en las formas de manejo convencional y mixta. Estos resultados coinciden con Camas et al. (2012) quienes aseguran que en los suelos utilizados de manera constante en monocultivo y sin ninguna práctica de conservación, son proclives a una mayor erosión, escurrimiento y como consecuencia mayor pérdida de minerales. Por ello, la disminución de la materia orgánica de manera sistemática, genera un reciclaje insuficiente de nutrientes en el suelo (Labrador, 2001; Figueroa et al., 2018; Barrales et al., 2020).

\section{CONCLUSIONES}

- Las evidencias que se presentan en este estudio indican que las características físicas y químicas son similares bajo los tres sistemas de manejo (convencional, agroecológico y mixto), esto se atribuye a una degradación generalizada del suelo por las diferentes prácticas antrópicas relacionadas con el cultivo de maíz y que son poco sostenibles.

- Desde el punto de vista biológico el sistema agroecológico mostró mayor abundancia y diversidad de microorganismos y macroorganismos, influenciado por un adecuado contenido de materia orgánica reportada para este sistema. Estos resultados indican un mejoramiento paulatino de la vida en el suelo por la incorporación de residuos de cosecha que practican los productores.

\section{DECLARACIÓN DE ÉTICA}

No aplicable.

\section{CONSENTIMIENTO PARA PUBLICACIÓN}

No aplicable.

\section{DISPONIBILIDAD DE DATOS}

Los conjuntos de datos utilizados o analizados durante el estudio actual están disponibles a solicitud razonable. Todos los datos generados o analizados durante este estudio se incluyen en este artículo publicado.

\section{CONFLICTO DE INTERESES}

Los autores declaran que no tienen intereses en competencia.

\section{FONDOS}

Los fondos utilizados para esta investigación fueron a través del Programa para el Desarrollo Profesional Docente (PRODEP).

\section{CONTRIBUCIÓN DE LOS AUTORES}

Conceptualización: Francisco Guevara-Hernández y Luis Alfredo Rodríguez-Larramendi. Metodología: Franklin B. Martínez-Aguilar y Carlos Ernesto AguilarJiménez. Software: Manuel Alejandro La O-Arias. Validación: Luis Alfredo Rodríguez-Larramendi. Análisis formal: Franklin B. Martínez-Aguilar y Manuel Alejandro La O-Arias. Investigación: Franklin B. Martínez-Aguilar, Francisco Guevara-Hernández, Carlos Ernesto Aguilar-Jiménez. Recursos: Luis Alfredo Rodríguez-Larramendi. Curación de datos: Mariela Beatriz Reyes-Sosa. Escritura: preparación del borrador original: Franklin B. Martínez-Aguilar y Luis Alfredo Rodríguez-Larramendi. Escritura: revisión y edición, Franklin B. Martínez-Aguilar, Luis Alfredo Rodríguez-Larramendi y Francisco GuevaraHernández. Visualización: Francisco GuevaraHernández. Supervisión: Carlos Ernesto Aguilar- 
Jiménez y Luis Alfredo Rodríguez-Larramendi. Administración del proyecto: Franklin B. MartínezAguilar y Francisco Guevara-Hernández. Adquisición de fondos: Mariela Beatriz Reyes-Sosa.

\section{AGRADECIMIENTOS}

Este artículo forma parte del proyecto de tesis doctoral del primer autor y es titulada "La sustentabilidad del agroecosistema maíz (Zea mays L.) en la región Frailesca, Chiapas. México". Se agradece a la Universidad Autónoma de Chiapas, por el apoyo y la oportunidad de realizar los estudios doctorales, al Programa para el Desarrollo Profesional Docente (PRODEP) por la beca otorgada y a todos los productores que proporcionaron información valiosa para el desarrollo de la presente investigación.

\section{LITERATURA CITADA}

Alcántar G., G., L. I. Trejo T. y F. C. Gómez M. 2016. Nutrición de cultivos. Colegio de Postgraduados. Montecillo, Texcoco, Estado de México. ISBN: 978-607-715-324-5.

Barrales-Brito, E., F. Paz-Pellat, J. D. Etchevers-Barra, C. Hidalgo-Moreno y A. Velázquez-Rodríguez. 2020. Dinámica de carbono en agregados del suelo con diferentes tipos de usos de suelo en el monte Tláloc, Estado de México. Terra Latinoamericana 38: 275-288. doi: https://doi.org/10.28940/ terra.v38i2.680.

Barrezueta U., S., A. Paz G. y J. Chabla C. 2017. Determinación de indicadores para calidad de suelos cultivados con cacao en provincia de el Oro-Ecuador. Rev. Cumbres 3: 17-24.

Bohn, H. B. Mcneal y G. O’Connor. 1993. Química del suelo. Limusa. México, D. F. ISBN: 968-18-4431-9.

Camas G., R., A. Turrent F., J. I. Cortes F., M. Livera M., A. González E., B. Villar S., J. López M., N. Espinosa P. y P. Cadena I. 2012. Erosión del suelo, escurrimiento y pérdida de nitrógeno y fósforo en laderas bajo diferentes sistemas de manejo en Chiapas, México. Rev. Mex. Cienc. Agríc. 3: 231-243.

Campbell, R. 2001. Ecología microbiana. Limusa. México, D. F. ISBN-13: 978-9681821104.

Cepeda D., J. M. 2016. Química de suelos. Trillas. México, D. F. ISBN-13: 978-968-24-4032-8.

Cleveland, C. C. and D. Liptin. 2007. C:N:P stoichiometry in soil: Is there a "Redfield ratio" for the microbial biomass? Biogeochemistry 85: 235-252. doi: https://doi.org/10.1007/ s10533-007-9132-0.

Correa, O. 2013. Los microorganismos del suelo y su rol indiscutido en la nutrición vegetal. pp. 1-11. In: M. DíazZorita, O. Correa, M. V. Fernández Canigia y R. S. Lavado (eds.). Aportes de la microbiología a la producción de los cultivos. Editorial de la Facultad de Agronomía. Buenos Aires, Argentina.
De la Rosa, I. N. y S. Negrete Y. 2012. Distribución espacial de la macrofauna edáfica en bosque mesófilo, bosque secundario y pastizal en la reserva La Cortadura, Coatepec, Veracruz, México. Rev. Mex. Biodiv. 83: 201-215.

Delgado-Ruiz, F., F. Guevara-Hernández y R. Acosta-Roca. 2018. Criterios campesinos para la selección de maíz (Zea mays L.) en Villaflores y Villa Corzo, Chiapas, México. CienciaUAT 13: 123-134. doi: https://doi.org/10.29059/cienciauat.v13i1.985.

Espinosa, J. y E. Molina. 1999. La acidez y encalado de suelos. International Plant Nutrition Institute. Quito, Ecuador.

Estrada H., I. R., C. Hidalgo M., R. Guzmán P., J. J. Almaraz S., H. Navarro G. y J. D. Etchevers B. 2017. Indicadores de calidad de suelo para evaluar su fertilidad. Agrociencia 51: 813-831.

Figueroa Jáuregui, M. L., M. R. Martínez Menez, C. A. Ortiz Solorio y D. S. Fernández Reynoso. 2018. Influencia de los factores formadores en las propiedades de los suelos en la Mixteca, Oaxaca, México. Terra Latinoamericana 36: 287299. doi: https://doi.org/10.28940/terra.v36i3.259.

García-Gutiérrez, C. y G. D. Rodríguez-Meza. 2012. Problemática y riesgo ambiental por el uso de plaguicidas en Sinaloa. Ra Ximhai 8: 1-10.

García, Y., W. Ramírez y S. Sánchez. 2012. Indicadores de la calidad de los suelos: Una nueva manera de evaluar este recurso. Pastos Forrajes 35: 125-138.

Guevara H., F., F. Delgado R., M. A. La O-Arias, L. A. Rodríguez L., R. Ortiz P., J. Á. Delgado R., J. A. Venegas V. y R. Pinto R. 2018. Análisis comparativo energético-económico del agroecosistema maíz bajo prácticas convencional y de conservación en la región Frailesca, Chiapas. México. Rev. Fac. Agron. (LUZ) 35: 343-364.

Guevara H., F., M. A. Hernández R., J. L. Basterrechea B., R. Pinto R., J. A. Venegas V., L. A. Rodríguez L. y P. Cadena I. 2019. Maíces locales; una contextualización de identidad tradicional. Rev. Fac. Cienc. Agrar. 51: 369-381.

INPOFOS (Instituto de la Potasa y el Fósforo). 1988. Manual internacional de fertilidad de los suelos. INPOFOS. Querétaro, Querétaro, México.

Jiménez-Ballesta, R., y V. González Quiñones. 2006. La calidad de suelos como medida para su conservación. Edafología 13: 125-138.

Jordán L., A. 2006. Manual de edafología. Departamento de Cristalografía, Mineralogía y Química Agrícola de la Universidad de Sevilla. Sevilla, España.

Labrador M., J. 2001. La materia orgánica en los agrosistemas. Mundi-Prensa. Madrid, España. ISBN: 9788449102486.

Lal, R. 1988. Effects of macrofauna on soil properties in tropical ecosystems. Agric. Ecosyst. Environ. 24: 101-116. doi: https:// doi.org/10.1016/0167-8809(88)90059-X.

López B., W., R. Reynoso S., J. López M., B. Villar S., R. Camas G. y J. O. García S. 2019. Caracterización físico-química de suelos cultivados con maíz en Villaflores, Chiapas. Rev. Mex. Cienc. Agríc. 10: 897-910. doi: https://doi.org/10.29312/ remexca.v10i4.1764.

López B., W., R. Reynoso S., J. López M., R. Camas G. y A. Tasistro. 2018. Diagnóstico de la compactación en suelos cultivados con maíz en la región Frailesca, Chiapas. Rev. Mex. Cienc. Agríc. 9: 897-910. doi: https://doi.org/10.29312/ remexca.v9i1.848. 
Martí, L., M. F. Filippini, A. Bermejillo, S. Troilo, C. Salcedo y A. Valdés. 2009. Monitoreo de cadmio y plomo en los principales fungicidas cúpricos comercializados en Mendoza, Argentina. Rev. FCA UNCuyo 41: 109-116.

Martínez B., F., F. Guevara, C. E. Aguilar, R. Pinto, M. A. La O, L. A. Rodríguez, and D. R. Aryal. 2020. Energy and economic efficiency of maize agroecosystem under three management strategies in the Frailesca, Chiapas (Mexico). 10: 81. doi: https://doi.org/10.3390/agriculture10030081.

Mendoza, R. B. y A. Espinosa. 2017. Guía técnica para muestreo de suelos. Universidad Nacional Agraria y Catholic Relief Services. Managua, Nicaragua.

Montiel, K. y M. Ibrahim. 2015. Manejo integrado de suelos para una agricultura resiliente al cambio climático. sistematización del ciclo de foros virtuales en el marco del Año Internacional de los Suelos (AIS) 2015. IICA. Guatemala, Guatemala.

Moreira, F. M. S., E. J. Huising y D. E. Bignell. 2012. Manual de biología de suelos tropicales. Muestreo y caracterización de la biodiversidad bajo suelo. Secretaria de Medio Ambiente y Recursos Naturales. México, D. F. ISBN: 9786077908319.

Morón, A., D. Martino y J. Sawchik. 1996. Manejo y fertilidad de suelos. Serie Técnica No. 76. INIA. Montevideo, Uruguay. ISBN: 9974 -38-063-4.

Mosquera, C. S., M. J. Martínez, J. A. Guerrero, E. W. Hansen. 2010. Caracterización estructural de la materia orgánica de tres suelos provenientes del Municipio de AquitaniaBoyacá, Colombia. Rev. Colomb. Quím. 39: 47-60.

Ortiz Solorio, C. A. 2019. Edafología. Trillas. Ciudad de México, México. ISBN: 978-607-17-3577-5.

Paredes M., M. y D. Espinosa V. 2009. Ácidos orgánicos producidos por rizobacterias que solubilizan fosfato: una revisión crítica. Terra Latinoamericana 28: 61-70

Pool-Novelo, L., A. Trinidad S., J. D. Etchevers B., J. Pérez M. y A. Martínez G. 2000. Mejoradores de la fertilidad del suelo en la agricultura de laderas de los altos de Chiapas, México. Agrociencia 34: 251-259.

Pool-Novelo, L., V. M. Kú-Quej, J. Chi-Quej y J. MendozaVega. 2019. Estimación del contenido de carbono orgánico en suelos y vegetación del estado de Campeche. Propuesta metodológica. Terra Latinoamericana 37: 317-328. doi: https://doi.org/10.28940/terra.v37i4.461.

Prieto M., J., F. Prieto G., O. A. Acevedo S. y M. A. Méndez M. 2013. Indicadores e índices de calidad de suelos (ICS) cebadores del sur del Estado de Hidalgo, México. Rev. Agron. Mesoam. 83-91.

Primavesi, A. 1980. Manejo ecológico del suelo. Ateneo. Sao Paulo, Brasil.

Pulleman, M., J. Hellin, D. Flores V. y W. López B. 2008. Calidad del suelo y rentabilidad de la finca: una situación en la que todos ganan. Leisa Rev. Agroecol. 24: 6-8.

Quiroga, A. R. y A. A. Bono. 2012. Manual de fertilidad y evaluación de suelos. INTA. La Palma, Argentina.
Rendón P., S., F. Artunduaga L., R. Ramírez P., J. A. Quiroz G. y E. I. Leiva R. 2011. Los macroinvertebrados como indicadores de la calidad del suelo en cultivos de mora, pasto y aguacate. Rev. Fac. Nac. Agr. Medellín 64: 5793-5802.

Rodríguez P., W., P. A. García R. y A. Fajardo O. 2016. Aplicaciones de técnicas espectroscópicas para el análisis de suelos. Rev. Fac. Cienc. Bás. 12: 228-251. doi: http:// dx.doi.org/10.18359/rfcb.2030.

Rodríguez S., F. 2005. Fertilizantes, nutrición vegetal. AGT. México, D. F. ISBN-13: 978-9684630079.

Rodríguez-Hernández, M. G., M. Á. Gallegos-Robles, L. Rodríguez- Sifuentes, M. Fortis-Hernández, J. G. LunaOrtega y U. González-Salas. 2020. Cepas nativas de Bacillus spp. como una alternativa sostenible en el rendimiento de forraje de maíz. Terra Latinoamericana 38: 313-321. doi: https://doi.org/10.28940/terra.v38i2.690.

SEMARNAT (Secretaría de Medio Ambiente y Recursos Naturales). 2002. NOM-021-RECNAT-2000 (Norma Oficial Mexicana). Que establece las especificaciones de fertilidad, salinidad y clasificación de suelos. Estudios, muestreo y análisis. SEMARNAT. México, D. F.

SEMARNAT (Secretaria del Medio Ambiente y Recursos Naturales). 2016. La degradación de los suelos en México. Los procesos que llevan a la desertificación. pp. 158-187. In: SEMARNAT. Informe de la situación del medio ambiente en México: compendio de estadísticas ambientales, indicadores clave de desempeño ambiental y de crecimiento verde. Edición 2015. SEMARNAT. Ciudad de México, México.

Shannon, C. E. and W. W. Weaver. 1949. The mathematical theory of community. University of Illinois Press, Urbana. Champaign, IL, USA.

SIAP (Servicio de Información agroalimentaria y pesquera). 2018. Atlas agroalimentario 2012-2018. SIAP, SAGARPA. CDMX, México.

Silva M., F. 2001. Fertilidad de suelos. Diagnóstico y control. Sociedad Colombiana de la Ciencia del Suelo. Guadalupe LTDA. Bogotá, Colombia. ISBN 958-95-299-1-7

Vallejo Q., V. E. 2013. Importancia y utilidad de la evaluación de la calidad de suelos mediante el componente microbiano. Experiencias en sistemas silvopastoriles. Colomb. For. 16: 83-99.

Vieira, M. J., B. Ochoa L., E. Sauër, M. Fischler y X. Marín. 1998. Manejo integrado de la fertilidad del suelo en zonas de ladera. Serie: Manejo de Tierras. Proyecto CENTA-FAOHolanda. El Salvador.

Wilson, M. G. 2017. Manual de indicadores de calidad del suelo para las eco regiones de Argentina. INTA. Buenos Aires, Argentina. ISBN: 978-987-521-826-0.

Yucra, S., M. Gasco, J. Rubio y G. F. Gonzales. 2008. Exposición ocupacional a Plomo y pesticidas órganofosforados: efecto sobre la salud reproductiva masculina. Rev. Perú Med. Exp. Salud Públ. 25: 394-402. 